\title{
Multi-terminal ballistic transport in one-dimensional wires
}

\author{
R. de Picciotto ${ }^{\mathrm{a}, *}$, H.L. Stormer ${ }^{\mathrm{a}, \mathrm{b}}$, A. Yacoby ${ }^{\mathrm{a}, \mathrm{c}}$, K.W. Baldwin ${ }^{\mathrm{a}}$, L.N. Pfeiffer ${ }^{\mathrm{a}}$, K.W. West ${ }^{\mathrm{a}}$

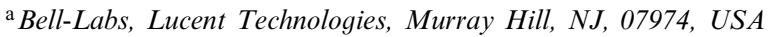 \\ ${ }^{\mathrm{b}}$ Dept. Phys. and Dept. Appl. Phys. Columbia University, New York, NY 10003, USA \\ ${ }^{\mathrm{c}}$ Braun Center for Submicron Research, Dept of Cond. Matter Physics, Weizmann Institute of Science, Rehovot 76100, Israel
}

\begin{abstract}
Recent experiments (Yacoby et al., Phys. Rev. Lett. 77 (1996) 4612; Solid State Commun. 101 (1997) 77; M. Rother et al., ICPS-24, Th-P137) have shown non-universal conductance quantization in one-dimensional wires that are fabricated using the cleaved edge overgrowth technique (L.N. Pfeiffer et al., Microelectronics J. 28 (1997) 817). In one of the papers (Yacoby et al., Phys. Rev. Lett. 77 (1996) 4612), it was speculated that the origin of the reduced conductance lies in the interface between the one-dimensional wire and the two-dimensional electron gas regions, which serve as ohmic contacts and thermal reservoirs. Here we report the results of a systematic study of such 2D-1D interfaces. By embedding a 2D-1D interaction section region of controllable length inside an otherwise isolated wire, we were able to study the properties of the coupling between these two subsystems. Our results show that 2D-1D interface is in fact the origin of the non-universal conductance. (C) 2000 Elsevier Science B.V. All rights reserved.
\end{abstract}

PACS: 73.20.Dx; 73.23.Ad; 73.23.Ps; 73.50.Jt

Keywords: One-dimensional wires; 2D-1D interface; Universal conductance

One-dimensional electronic systems, the so-called Luttinger-liquids, are expected to show unique transport properties as a consequence of the Coulomb interaction between carriers [1-7]. In contrast to Fermi liquid theory [8-12], the Luttinger model predicts that even the weakest impurity embedded in an otherwise perfectly clean 1D wire will completely suppress its conductance at zero temperature. Furthermore, the tunneling conductance into such a perfect wire will also be completely suppressed at zero temperature.

* Corresponding author.
One of the fingerprints of a clean non-interacting $1 \mathrm{D}$ conductor is its quantized conductance in multiples of the universal value $g_{0}=2 e^{2} / h$. This quantization results from an exact cancellation of the increasing electron velocity and the decreasing density of states as the carrier density increases [13-16]. Therefore, as subsequent $1 \mathrm{D}$ electronic subbands are filled with electrons the conductance increases in a series of plateaus (or steps) with values equal to $g_{0}$ multiplied by the number of occupied wire modes. Surprisingly, the inclusion of interactions does not alter this prediction. Early papers [4-7], considering infinitely long wires, did in fact predict quantization 
of conductance with a renormalized, non-universal, value of $g=v \cdot g_{0}$, where $v=\left(1+U / E_{\mathrm{F}}\right)^{-1 / 2}$. Here, $U$ is the strength of the Coulomb interaction between neighboring electrons and $E_{\mathrm{F}}$ is the Fermi energy. Later works [17-19], however, considered a more realistic wire of finite length that is connected to large, Fermi-liquid like, reservoirs at both ends. In the clean limit, the contact resistance to the reservoirs dominates the conductance and the universal value, $g_{0}$, is restored. Oreg and Finkelstein [20] have further demonstrated that if one calculates the conductance due to the self-consistent electric field in the wire rather than the external electric field, the universal value is restored even for the infinite wire case. In contrast to these theoretical predictions, recent papers [21-23], using wires that were fabricated by the cleaved edge overgrowth method [24] showed that the contribution of each one-dimensional channel to the overall conductance is not universal and smaller than the conductance quantum. This non-universal conductance is measured with short wires, much shorter than the 1D back-scattering length. In these two-terminal measurements, however, the combined contribution of the one-dimensional channel and the unavoidable interfaces between this channel and the 2D reservoirs at its two ends was determined. Here we present results of measurements designed to address the origin of this puzzling observation. We embedded a 2D-1D interaction region (IR) of controllable length within an otherwise isolated $1 \mathrm{D}$ wire in order to study the properties of the coupling between these two subsystems.

The geometry of the devices under study is illustrated in Fig. 1. A substrate, containing a two-dimensional electron gas (2DEG) in a single-side doped GaAs quantum well is epitaxially grown on a [ 1000$]$ GaAs surface. The 2DEG has a carrier density $n_{\mathrm{s}} \sim 2.5 \times 10^{11} \mathrm{~cm}^{-2}$, and a mobility of $\sim 4 \times 10^{6} \mathrm{~cm}^{2} / \mathrm{V} \mathrm{s}$. The substrate is then cleaved in situ and a 2 nd modulation doping sequence is overgrown on the [0 111$]$ cleaved edge. This procedure results in high-quality one-dimensional (1D) electronic channels propagating along the cleaved edge. These channels, existing throughout one cleaved edge of the 2D plane, are coupled to the adjacent 2DEG (see Fig. 1b).

The length of a cleaved edge overgrowth wire $(\mathrm{CEO}-\mathrm{W})$ that is measured is defined by a gate which is pre-patterned on the top surface of the structure

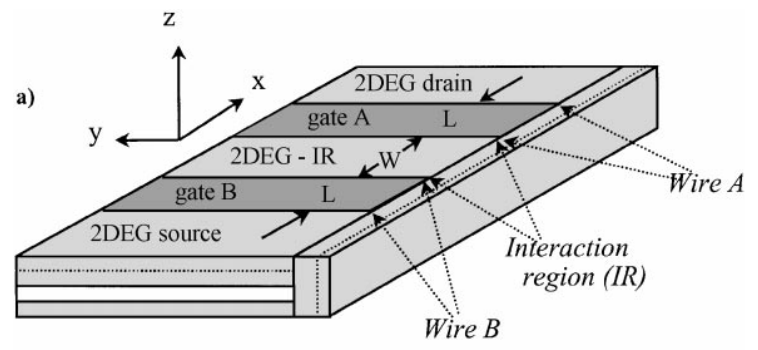

b)

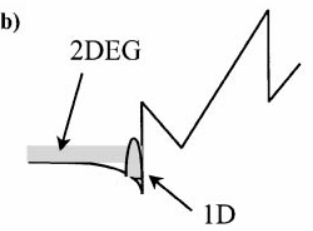

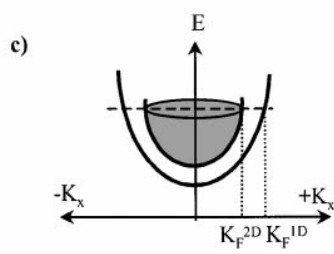

Fig. 1. (a) The geometry of the device under study (see text) (b) Conduction band profile in the $\mathrm{Y}$ direction at the quantum well level and in a non-gated region. The 2DEG and the first 1D mode are also shown. (c) Dispersion relation for electrons in the 2DEG and 1D wire. The Fermi wavevector of the two systems is indicated.

prior to the cleave and overgrowth steps (see Fig. 1a). A negative voltage applied to this gate depletes the 2DEG from underneath it, leaving only the wire to connect the two 2DEG sections. Thus, the width of the gate defines a length, $L$, over which the $1 \mathrm{D}$ wire is isolated from the adjacent 2DEG. By increasing the voltage beyond depletion of the 2DEG one can control the number of occupied 1D modes in this region.

To study the interaction of current carriers in a wire with the adjacent 2DEG we split the gate in two (gates A and B in Fig. 1a), creating an 2D-1D overlap region of controllable length, $W$, between the wire and a 2DEG. With this device, there are three distinct regions along the wire: In the two long sections $|x|>W / 2+L$ the wire is coupled to the adjacent 2 DEGs. These regions are the source and drain. The two regions $W / 2<|x|<W / 2+L$ are isolated wires. The number of channels in each wire is controlled independently by gates $\mathrm{A}$ and $\mathrm{B}$. The region $|x|<W / 2$ is the interaction region (IR). Here $1 \mathrm{D}$ modes are coupled to the 2DEG between the two gates. Current is injected from the source into an isolated wire of length $L$, electrons are then allowed to interact with an adjacent, floating, 2DEG in the IR for a length $W$ before proceeding into another wire and finally into the drain. Since the gates have an electrostatic influence 


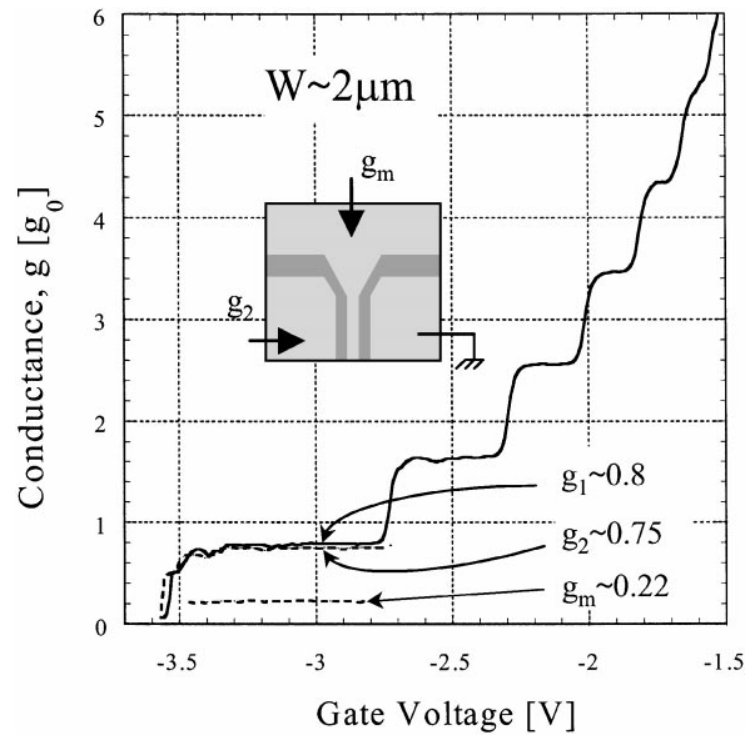

Fig. 2. Conductance, in units of the conductance quantum $2 e^{2} / h$, of an isolated $2 \mu \mathrm{m}$ long wire, $g_{1}$ (solid line) and the conductance of two isolated single mode wires combined in series with a $W \sim 2 \mu \mathrm{m}$ long interaction region, $g_{2}$, (dashed line) plotted against the applied gate voltage. The conductance from the interaction reagion into both single mode wires, $g_{m}$, is also shown (dashed line). Inset: Graphical illustration of the measurement scheme.

on the wire, the potential along the wire is lower in the IR - where the gate is absent. The electrons thus also interact with the smooth resultant static 1D potential dip of length $W$. While this trough is estimated to be deep enough to change the number of $1 \mathrm{D}$ channels in the IR, it has a smooth shape (due to a large distance of $\sim 0.5 \mu \mathrm{m}$ between the gates and the wire). The overall influence of this electrostatic potential on back scattering in our device is thus minor.

The behavior of such a device is shown in Fig. 2 . The conductance of each individual $L \sim 2 \mu \mathrm{m}$ long wire, $g_{1}$, when the other gate is not activated, exhibits clear conductance plateaus as a function of gate voltage with a plateau height of $g_{1} \sim 0.8 g_{0}$, as shown by the solid line in this figure. With both gates activated, we measure the overall conductance, $g_{2}$, of the two single mode isolated wires plus the IR. For a relatively long IR, of $W \sim 10 \mu \mathrm{m}, g_{2}$ equals half the conductance of each individual gatewire; $g_{2} \sim 0.4 g_{0} \sim \frac{1}{2} g_{1}$ (not shown). This indicates complete thermalization in the IR, leading to ohmic combination of the two resistors in series, resulting in half the conductance for the combined system.

The same measurement with a shorter IR, $W \sim$ $2 \mu \mathrm{m}$, renders a very different result, as shown in Fig. 2. Under the same conditions, namely when both isolated wires support a single mode, the overall conductance nearly equals the conductance of each isolated wire in spite of the presence of the IR $\left(g_{2} \sim\right.$ $0.75 g_{0} \sim 0.94 g_{1}$ at a temperature, $\theta=300 \mathrm{mK}$ ). This clearly demonstrates ballistic 1D transport through a $2 \mu \mathrm{m}$ long IR where the wire overlaps with a $2 \mathrm{DEG}$. For an intermediate IR length of $W \sim 6 \mu \mathrm{m}$ we find $g_{2} \sim 0.6 g_{0} \sim 0.75 g_{1}$ (not shown) - indicating partial back scattering.

The conductance from the 2DEG of the IR into the single mode wires on both sides, $g_{m}$, equals $2 g_{1}$ for $W \sim 10 \mu \mathrm{m}$ (not shown). This result is again the ohmic combination of the two wires - now in parallel - resulting in twice the conductance. For $W \sim 2 \mu \mathrm{m}$, the value of the measured conductance, $g_{m}$, (again when both wires support only one mode) is much smaller; $g_{m} \sim 0.22 g_{1}$ (see Fig. 1 ).

We model the behavior of our device within a Buttiker-Landauer scattering approach. The coupling of the 2DEG to the wire at the drain and source regions, $|x|>L+W / 2$, is modeled by assuming a maximal emissivity $\alpha=g_{1} / g_{0} \sim 0.8$. The shorter length of the IR is modeled by a smaller emissivity $-T_{1} \alpha$. Here $T_{1}(W)$ is the transmission probability from the 2DEG into each branch of the 1D dispersion of the first channel $\left( \pm K_{\mathrm{F}}^{1 \mathrm{D}}\right)$. Assuming symmetric coupling of the 2DEG to both sides, the scattering matrix of the junction may be written in terms of $\alpha$ and $T_{1}$. One finds for the measured quantities; $g_{2}=\alpha\left(1-\frac{1}{2} T_{1}\right) g_{0}$ and $g_{m}=2 T_{1} \alpha g_{0}$. The two measured values are both in excellent agreement with this model using $T_{1} \sim$ 0.13 for $W \sim 2 \mu \mathrm{m}$. The value of $\alpha$ is deduced from $g_{1}$, which is measured independently.

The reduction of $g_{2}$ compared to $g_{1}$ is a consequence of $1 \mathrm{D}$ backscattering that is mediated by the $2 \mathrm{DEG}$ in the IR. An electron in the wire that was scattered into the $2 \mathrm{DEG}$ has an equal probability to scatter back into either direction in the $1 \mathrm{D}\left( \pm K_{\mathrm{F}}^{1 \mathrm{D}}\right)$ therefore loses on average all its momentum (assuming fast relaxation in the 2DEG). At first glance it would seem that $1 \mathrm{D}$ backscattering might be modified by the presence of the 2DEG even without charge transfer between the two subsystems. However, e-e scattering that 
involves momentum exchange without charge transfer can not occur because the 2D Fermi momentum, $k_{\mathrm{F}}^{2 \mathrm{D}}$, is smaller than the one in the $1 \mathrm{D}$ wire (see Fig. 1b and $\mathrm{c}$ ). This excludes this scattering mechanism to first order. The smallness of $k_{\mathrm{F}}^{2 \mathrm{D}}$ also leads to poor screening of the disorder potential at wave vectors relevant to $1 \mathrm{D}$ back scattering. Therefore, the additional back scattering induced by the IR is mostly due to transmission charge transfer between the two subsystems. Our results thus indicate a mean free path for scattering between the 2DEG and the 1D, $l_{2 \mathrm{D} \leftrightarrow 1 \mathrm{D}} \sim 6 \mu \mathrm{m}$ ( for $W \sim 6 \mu \mathrm{m} T_{1} \sim \frac{1}{2}$ ).

Since this new length scale is comparable to the back scattering length inside a CEO-W, an analysis of the 2D-1D interface is called for - as was suggested in previous work [21]. Using a Boltzmann equation approach, the authors derived an expression for the conductance of an ideal, single channel, wire which is coupled to 2DEGs at its ends; $g_{1}=g_{0}(1+$ $\left.2 l_{2 \mathrm{D} \leftrightarrow 1 \mathrm{D}} / l_{\mathrm{B}}\right)^{-1 / 2}$, where $l_{\mathrm{B}}$ is the $1 \mathrm{D}$ back-scattering length at the two ends, namely in the $2 \mathrm{D}-1 \mathrm{D}$ overlaps region. In view of the above discussion, we assume that $l_{\mathrm{B}}$ is not very different from the back scattering length inside an isolated CEO-W, which was previously found to be $\sim 20 \mu \mathrm{m}$ [21]. One finds an expected conductance of $g_{1} \sim 0.79 g_{0}-$ in excellent agreement with the observed value.

In conclusion, we have shown results of a systematic study of CEO-W 2D-1D interface. By embedding a 2D-1D overlap section of controllable length inside an isolated wire, we were able to study the properties of the coupling between the two systems. We find a scattering mean free path of $\sim 6 \mu \mathrm{m}$. Our results show that the origin of the non-universal conductance [21] lies in the 2D-1D interface.

\section{References}

[1] J.T. Devreese (Ed.), Highly Conducting One Dimensional Solids, Plenum, New York, 1979.

[2] D.C. Mattis, The Many Body Problem, World Scientific, Singapore, 1992.

[3] F.D.M. Haldane, J. Phys. C 14 (1981) 2585.

[4] W. Apel, T.M. Rice, Phys. Rev. B 26 (1992) 7063.

[5] C.L. Kane, M.P.A. Fisher, Phys. Rev. Lett. 68 (1992) 1220.

[6] D. Yue et al., Phys. Rev. B 49 (1994) 1966.

[7] M. Ogata, H. Fukuyama, Phys. Rev. Lett. 73 (1994) 468

[8] A.V. Chaplik, Zh. Exsp. Teor. Fiz. 60 (1971) 1845.

[9] H.F. Fukuyama, E. Abrahams, Phys. Rev. B 27 (1983) 5976.

[10] G.F. Guliani, J.J. Quinn, Phys. Rev. B 26 (1982) 4421.

[11] T. Jungwirth, A.M. Macdonald, Phys. Rev. B 53 (1996) 7403.

[12] B.L. Altshuler, A.G. Aronov, in: A.L. Efros, M. Pollak (Eds.), Electron-Electron Interactions in Disordered Systems.

[13] R. Landauer, IBM J. Res. Dev. 32 (1988) 306.

[14] M. Buttiker, Phys. Rev. Lett. 57 (1996) 1761.

[15] C.W.J. Beenakker, H. von Houten, in: H. Ehrenreich, D. Turnbull (Eds.), Solid State Physics, Semiconductor Heterostructures and Nanostructures, Academic Press, New York, 1991.

[16] Y. Imry, Introduction to Mesoscopic Physics, Oxford University, Oxford, 1997.

[17] I. Safi, H.J. Schulz, Phys. Rev. B 52 (1995) R 17040.

[18] V.V. Ponomarenko, Phys. Rev. B 52 (1995) R 8666.

[19] D.L. Maslov, Phys. Rev. B 52 (1995) R 14368.

[20] Y. Oreg, A.M. Finkel'stein, Phys. Rev. B 54 (1996) 14255.

[21] A. Yacoby et al., Phys. Rev. Lett. 77 (1996) 4612.

[22] A. Yacoby et al., Solid State Comm. 101 (1997) 77.

[23] M. Rother et al., ICPS-24, Th-P137.

[24] L.N. Pfeiffer et al., Microelectronics J. 28 (1997) 817. 